\title{
Investigation of plasma asprosin and saliva levels in newly diagnosed type 2 diabetes mellitus patients treated with metformin
}

\author{
Nevzat Gozel', Faruk Kilinc ${ }^{2}$ \\ ${ }^{1}$ Department of Internal Medicine, Faculty of Medicine, Firat University, Elazig, Turkey \\ ${ }^{2}$ Department of Endocrinology, Faculty of Medicine, Firat University, Elazig, Turkey
}

\begin{abstract}
Introduction: Asprosin is a hormone that was first reported by Romere et al. [2016]. Its secretion is induced in the case of starvation. Asprosin promotes hepatic glucose release. There is no literature information available in humans to demonstrate how blood and saliva asprosin levels of patients with the newly identified type 2 diabetes mellitus (T2DM) changed after metformin treatment. We aim to examine these changes and contribute to the literature in this sense.

Material and methods: A total of 60 individuals: 30 healthy volunteers and 30 newly identified cases of T2DM whose treatment had been initiated, were included in the investigation. Blood and saliva sample specimens were carefully taken from both groups. Saliva asprosin and serum levels were tested using the ELISA method. Immunohistochemical methods were used to test asprosin formation sites in salivary gland tissues.

Results: Similarly increased asprosin levels were observed in patients from the newly diagnosed T2DM group compared with the healthy control group $(\mathrm{p}=0.003)$. In the newly defined T2DM group, blood asprosin levels decreased significantly after three months of metformin treatment $(p=0.032)$. In terms of saliva asprosin levels, when the healthy control group and the newly identified T2DM group were compared, saliva asprosin levels were found to be higher in the newly identified T2DM group $(\mathrm{p}<0.001)$. With immunohistochemical staining, asprosin immunoreactivity was observed in the submandibular and parotid glands.

Conclusions: In our study, serum and saliva asprosin levels increased significantly in the newly identified individuals with type 2 diabetes, which suggests that asprosin could form a critical risk related to T2DM. Higher asprosin levels are an important marker for predicting diabetes development, and that this hormone can be signified as a main or target molecule in the treatment of diabetes. (Endokrynol Pol 2021; 72 (1): 37-43)
\end{abstract}

Key words: type 2 diabetes mellitus; asprosin; metformin

\section{Introduction}

Diabetes, beyond glycaemic control, is a complex and chronic disease, the aetiology of which is multifactorial, requiring continuous medical care [1]. There are four major types of diabetes: type 1 diabetes mellitus (T1DM), type 2 diabetes mellitus (T2DM), gestational diabetes mellitus (GDM), and others [2]. As of 2019, more than 463 million people are estimated to live with diabetes worldwide, and according to the 2019 estimates of this figure, the International Diabetes Federation has increased the prediction to 578.4 million by 2030; it predicts that it will reach 700.2 million by 2045 [3]. Among these T2DM is the largest spread with more than $90 \%$ of patients [4]. Beta-cell dysfunction or an increased hepatic glucose output and insulin resistance are important pathophysiological features of T2DM [5].

Various antidiabetic drugs, like metformin and glucagon-like peptide 1 (GLP-1) receptor analogues, show their effects by partially reducing hepatic glucose output $[4,6]$. Except under specific conditions, usually metformin is prescribed as the first drug for the treatment of hyperglycaemia in T2DM patients. When identified with diabetes, metformin therapy can be initiated simultaneously with lifestyle change recommendations $[7,8]$. Hepatic glucose suppression appears as a better treatment agent for T2DM.

Asprosin is a protein consisting of 140 amino acids with a C-terminal region cleaved with pro brillin [9]. It is secreted from white adipose tissue, and its secretion is induced in the case of starvation. Asprosin cAMP (G protein-cyclic adenosine monophosphate) - protein kinase promotes hepatic glucose production via the A pathway and thus has a protective effect from hypoglycaemia $[9,10]$. However, studies with animal models have shown that asprosin administration causes hyperglycaemia and hyperinsulinaemia [9]. In animal models and studies in humans, insulin resistance and 
serum asprosin levels have been reported to increase in T2DM patients [11]. In another study, it has been shown that insulin sensitivity increases as a result of the neutralisation of asprosin by specific antibodies [9]. Considering all this information, reducing asprosin activity might be considered as a new therapeutic potential to treat the diseases related to insulin resistance: obesity, gestational diabetes, polycystic ovary syndrome, and especially T2DM. Some studies show a relationship between serum asprosin and T2DM in humans. However, there is no literature information showing the blood and saliva asprosin levels of these (the newly identified T2DM) patients and how these levels change after metformin treatment. Also, there has been no study yet evaluating the effects of metformin, an oral antidiabetic that decreases hepatic glucose output, and asprosin, which has a diabetogenic effect by increasing hepatic glucose output. Our aim in the study is to examine these changes and contribute to the literature in this sense.

\section{Material and methods}

\section{Participants}

Every care was taken to obtain ethical approval from Firat University Non-Interventional Research Ethics Committee before the study with the decision dated 24/01/2019 and numbered 97132852/050.01.04/02/16. A total of 60 individuals: 30 healthy volunteers and 30 the newly identified cases of T2DM, participated in the study. Diabetes identification was placed as per the identification/diagnostic criteria of the American Diabetes Association (ADA) [12]. Accordingly, after eight hours of fasting, fasting blood glucose $\geq 126 \mathrm{mg} / \mathrm{dL}, 75 \mathrm{~g}$ of oral glucose tolerance test (OGTT) second-hour blood glucose $\geq 200 \mathrm{mg} / \mathrm{dL}$, haemoglobin $\mathrm{A}_{1 c}\left(\mathrm{HbA}_{1 c}\right)$ $\geq 6.5 \%$ or randomly observed individuals with symptoms of hyperglycaemia, whose blood sugar was measured as $\geq 200 \mathrm{mg} / \mathrm{dL}$, were considered T2DM. [12]. Metformin treatment was started in the newly diagnosed T2DM patients at a dose of $2000 \mathrm{mg} /$ day and continued for three months. Individuals with a history of diabetes, hypertension, any known cardiovascular disease, malignancy, use of immunosuppressive drugs, steroids, or acute/chronic liver disease, acute/chronic kidney failure, infection, and pregnant or mentally retarded patients with socio-cultural communication problems were excluded and did not participate in the study.

\section{Sample specimens}

Fasting plasma glucose, low-density lipoprotein cholesterol (LDLC), triglyceride, alanine aminotransferase (ALT), aspartate aminotransferase (AST), urea, creatinine, and complete blood count (CBC) values were examined in both the T2DM group and the healthy control group. To better evaluate the glycaemic status in the T2DM group, satiety plasma glucose and $\mathrm{HbA1c}$ levels were also evaluated. Parameters such as ALT, AST, LDL, triglyceride, urea, creatinine, and fasting and postprandial blood glucose levels were carefully measured using an Olympus AU 600 (Olympus Optical Co. Ltd, Tokyo, Japan) automated analyser using commercially available Olympus kits. Haemoglobin, haematocrit, and $\mathrm{HbA}_{1 c}$ levels were measured with a CELL-DYN 3700 (USA) device.

At the time of admission, a $4 \mathrm{ml}$ blood sample specimen and $4 \mathrm{~mL}$ saliva sample specimen from the left antecubital region in the healthy control group and the patients were taken into tubes containing aprotinin (protease inhibitor). The blood sample speci- mens were treated in the tube with EDTA, shaking gently on ice batteries without wasting time, and centrifuged at $4000 \mathrm{rpm}$ for 10 minutes, then placed in Eppendorf tubes followed by storing at $-80^{\circ} \mathrm{C}$ until carrying out the study. The saliva sample specimens were transferred to aprotinin tubes and centrifuged under the same conditions and kept at $-80^{\circ} \mathrm{C}$ until the working day. Asprosin levels in serum and saliva sample specimens, Human (asprosin); catalogue number: 201-12-7193 (Sunred Biological Technology Co. Ltd, Shanghai, CHINA) was studied by ELISA method following the working techniques as mentioned in the kit catalogues. Measuring range of Human Asprosin ELISA kit: 1-300 ng/mL, intra-assay: $\mathrm{CV}$ value $<10 \%$, inter-assay: $\mathrm{CV}$ value $<12 \%$, and sensitivity was $0.756 \mathrm{ng} / \mathrm{mL}$. An automatic washer Bio-Tek ELX50 (BioTek Instruments, USA) was used for plate washings, and ChroMate Microplate Reader P4300 device (Awareness Technology Instruments, USA) was used for absorbance readings. Test results are given in $\mathrm{ng} / \mathrm{mL}$. The asprosin kit has been shown to measure asprosin in saliva sample specimens [13]. We also measured the saliva sample specimens according to this method.

\section{Immunohistochemistry}

Submandibular and parotid glands from salivary glands were used for asprosin immunoreactivity. Archived submandibular and parotid gland tissue samples of five patients (were not diagnosed), and five T2DM patients who had provided submandibular and parotid gland tissue samples due to diagnostic purposes were included in the study. Sections taken from paraffin blocks with a thickness of 5-6 mm were placed on PolySync slides. The deparaffinised tissues were treated with graded alcohol series using boiled citrate buffer to retrieve antigen at $\mathrm{pH} 6.0$ in a microwave oven $(750 \mathrm{~W})$ for $7+5$ minutes. These tissues were cooled at $24^{\circ} \mathrm{C}$ for $\sim 20$ minutes after boiling and were washed with PBS (Phosphate Buffered Saline, P4417, Sigma-Aldrich, USA) for $3 \times 5$ minutes, followed by incubation on hydrogen peroxide block solution for five minutes to avoid endogenous peroxidase activity (Hydrogen Peroxide Block, TA-125-HP, Lab Vision Corporation, USA). Five-minute Ultra V Block (TA-125-UB, Lab Vision Corporation, USA) solution was applied to these tissues after they were rinsed with PBS for $3 \times 5$ minutes to prevent ground paint followed by continuous incubation at $24^{\circ} \mathrm{C}$ for 1 hour in a moist environment containing primary antibody (Polyclonal Antibody to Asprosin, PAA332Hu01, Cloud-Clone Corp., USA) diluted by $1 / 200$. Tissues were washed $3 \times 5$ minutes with PBS after administration of primary antibody and were incubated for 30 minutes at room temperature in a moist environment Along with secondry antibody (biotinylated Goat Anti-Pollivalent [anti-mouse/rabbit IgG], TP-125-BN, Lab Vision Corporation, USA). Tissues were taken into phosphate buffered saline (PBS) after washing for $3 \times 5$ minutes with PBS and incubating with Streptavidin Peroxidase (TS-125-HR, Lab Vision Corporation, USA) at room temperature for 30 minutes after secondary antibody administration. After dropping the 3-amino-9-ethyl carbazole (AEC) substrate + AEC Chromogen (AEC Substrate, TA-015 and HAS, AEC Chromogen, TA-002-HAC, Lab Vision Corporation, USA) solution into the tissues and after an image signal was obtained in the light microscope, washing with PBS was applied simultaneously. Tissues were then contrasted using Mayer's haematoxylin by treating with PBS + distilled water and stopped with suitable closure solution (Large Volume Vision Mount, TA-125-UG, Lab Vision Corporation, USA). The preparations were examined using a Leica DM500 microscope and photographed (Leica DFC295).

\section{Statistical analysis}

The statistical analyses were carried out with SPSS 24 (Statistical Package for the Social Sciences version 22.0 (SPSS Inc., Chicago, USA). The experimental data were subjected to variance analysis, Student's t-test, descriptive statistical methods, and equivalents. Analysis of covariance (ANCOVA) model plus post hoc t-test with Bonferroni's correction was used to compare levels of blood and 
saliva asprosin among the groups adjusting for age. In our study, statistical significance was accepted as $\mathrm{p}<0.05$.

\section{Results}

The clinical, demographic, and laboratory data of the individuals included in the study are summarised in Table 1 . The average age of the diabetic patient group was remarkably high compared to the patients in the healthy control group ( $\mathrm{p}<0.001)$. In the T2DM group and the healthy control group, the ratio of male to female was balanced. Blood sugar levels during fasting were higher in the specific group patients having diabetes $(p<0.001)$. No significant variation was noted between the group patients considering the tests based on liver functions. While LDL levels were significantly higher in patients with diabetes compared to the healthy control group at the time of the first identification $(124.2 \pm 31.9 ; 152.03 \pm 51.3, p=0.01$, respectively), the statistical variations between the group patients disappeared after three months of metformin treatment (respectively, $124.2 \pm 31.9 ; 127.9 \pm 33.6, \mathrm{p}=0.67$ ). Blood asprosin levels were measured, respectively, in the healthy control group, during the identification of patients with type 2 diabetes, and after three months of treatment as $25.16 \pm 14.49,49.52 \pm 41.98$, and $27.27 \pm 19.77 \mathrm{ng} / \mathrm{mL}$ (Tab. 1). In terms of blood asprosin levels, when the healthy control group patients and the newly identified T2DM group patients was compared, the blood asprosin levels were identified statistically higher in the newly identified T2DM group ( $p=0.003)$. After age adjustment and Bonferroni correction, the blood asprosin levels were higher in the newly identified T2DM group compared to the healthy control group patients $(\mathrm{p}=0.04)$. In the newly identified T2DM group, blood asprosin levels decreased significantly after three months of metformin treatment $(\mathrm{p}=0.032)$. When the control group and the three-month treatment group were compared for blood asprosin levels the differences were statistically non-significant $(p=0.954)$.

Saliva asprosin levels are measured respectively in the healthy control group patients, during the identification of patients with type 2 diabetes and after 3 months of treatment as $23.79 \pm 10.27,45.85 \pm 23.07$, $34.41 \pm 19.02 \mathrm{ng} / \mathrm{mL}$ (Tab. 1, Fig. 2). In terms of saliva asprosin levels, when the healthy control group patients and the newly identified T2DM group patients were compared, in the newly identified T2DM group, asprosin levels were statistically significant higher $(\mathrm{p}<0.001)$. The saliva asprosin levels were higher in

Table 1. Clinical, demographic, and laboratory data of the groups included in the study

\begin{tabular}{|c|c|c|c|c|c|c|}
\hline & $\begin{array}{l}\text { Control } \\
(\mathrm{n}=\mathbf{3 0})\end{array}$ & $\begin{array}{c}\text { DM } \\
\text { 0. month } \\
(\mathrm{n}=30)\end{array}$ & $\begin{array}{c}\text { DM } \\
\text { 3. month } \\
(n=30)\end{array}$ & P1 & P2 & P3 \\
\hline Age (Years) & $30.10 \pm 8.8$ & $54.63 \pm 10.8$ & $54.63 \pm 10.8$ & $<0.001$ & $<0.001$ & $>0.05$ \\
\hline Gender (Male/Female) & $16 / 14$ & $17 / 13$ & $17 / 13$ & $>0.05$ & - & - \\
\hline Systolic BP [mm Hg] & $105 \pm 20.2$ & $119 \pm 17.5$ & $111.1 \pm 12.4$ & $<0.05$ & - & - \\
\hline Diastolic BP [mm Hg] & $67 \pm 7.2$ & $76.0 \pm 13.2$ & $76 \pm 9.3$ & $<0.05$ & - & - \\
\hline BMI $\left[\mathrm{kg} / \mathrm{m}^{2}\right]$ & $23.6 \pm 4.8$ & $26.4 \pm 3.6$ & $25.3 \pm 4.9$ & $>0.05$ & $>0.05$ & $>0.05$ \\
\hline FPG [mg/dL] & $89.9 \pm 10.7$ & $178.3 \pm 47.10$ & $136.10 \pm 39.3$ & 0.001 & 0.001 & 0.001 \\
\hline PPG [mg/dL] & & $287 \pm 84.9$ & $217.17 \pm 77.28$ & - & - & 0.001 \\
\hline AST [U/L] & $20.9 \pm 11.06$ & $23.3 \pm 13.2$ & $21.40 \pm 8.93$ & 0.45 & 0.09 & 0.22 \\
\hline ALT [UL] & $22.6 \pm 14.10$ & $29.4 \pm 17.8$ & $21.90 \pm 13.09$ & 0.10 & 0.82 & 0.084 \\
\hline Urea $[\mathrm{mg} / \mathrm{dL}]$ & $25.07 \pm 7.9$ & $29.47 \pm 8.1$ & $31.37 \pm 10.73$ & 0.03 & 0.01 & 0.144 \\
\hline Creatinine $[\mathrm{mg} / \mathrm{dL}]$ & $0.67 \pm 0.16$ & $0.75 \pm 0.24$ & $0.75 \pm 0.23$ & 0.18 & 0.17 & 0.973 \\
\hline Haemoglobin [gr/dL] & $12.7 \pm 1.5$ & $14.2 \pm 1.78$ & $14.11 \pm 1.81$ & $<0.01$ & $<0.01$ & $>0.05$ \\
\hline Haematocrit (\%) & $39.2 \pm 3.85$ & $43 \pm 5.2$ & $42.3 \pm 4.95$ & 0.001 & 0.008 & 0.310 \\
\hline LDL-C [mg/dL] & $124.2 \pm 31.90$ & $152.03 \pm 51.3$ & $127.9 \pm 33.6$ & 0.01 & 0.67 & 0.025 \\
\hline Triglycerides [mg/dL] & $204.8 \pm 80.126$ & $255.9 \pm 150.78$ & $208.9 \pm 84.2$ & 0.10 & 0.84 & 0.04 \\
\hline $\mathrm{HbA}_{1 \mathrm{c}}(\%)$ & & $7.5 \pm 1.6$ & $6.2 \pm 1.2$ & - & - & 0.001 \\
\hline Asprosin plasma [ng/mL] & $25.16 \pm 14.49$ & $49.52 \pm 41.98$ & $27.27 \pm 19.77$ & 0.003 & 0.954 & 0.032 \\
\hline Asprosin saliva [ng/mL] & $23.79 \pm 10.27$ & $45.85 \pm 23.07$ & $34.41 \pm 19.02$ & $<0.001$ & 0.068 & $<0.001$ \\
\hline
\end{tabular}

P1 — control with DM-0 month comparison; P2 — control with DM-3 months comparison; P3 — DM0. months with DM-3 months comparison; BP — blood pressure; $\mathrm{BMI}$ — body mass index; FPG — fasting plasma glucose; PPG — postprandial blood glucose; AST — aspartate aminotransferase; ALT — alanine aminotransferase; LDL-C — low-density lipoprotein cholesterol; $\mathrm{HbA}_{1 \mathrm{c}}$ — haemoglobin $\mathrm{A}_{1 \mathrm{c}}$ 


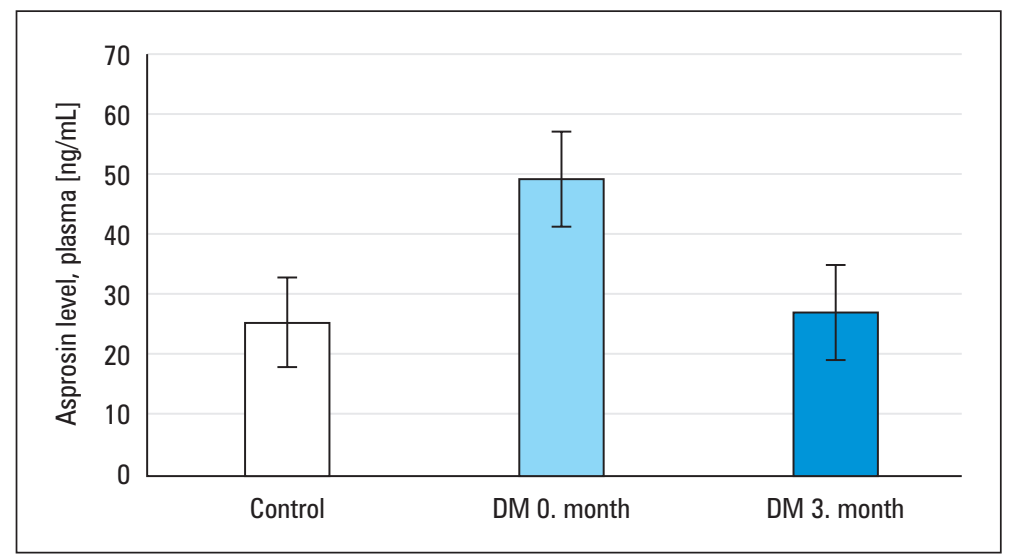

Figure 1. Asprosin levels in the plasma of all three groups. DM — diabetes mellitus

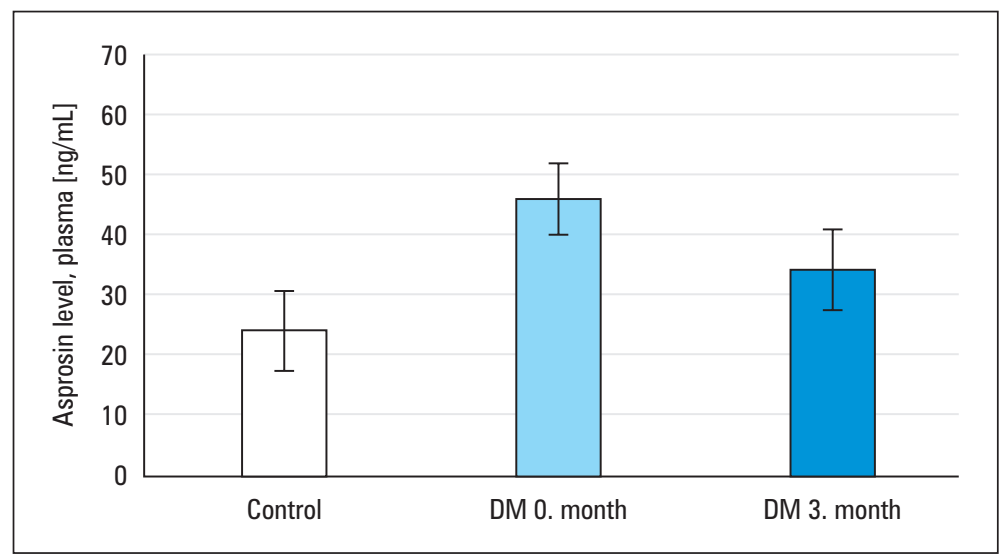

Figure 2. Asprosin levels in the saliva of all three groups. DM - diabetes mellitus
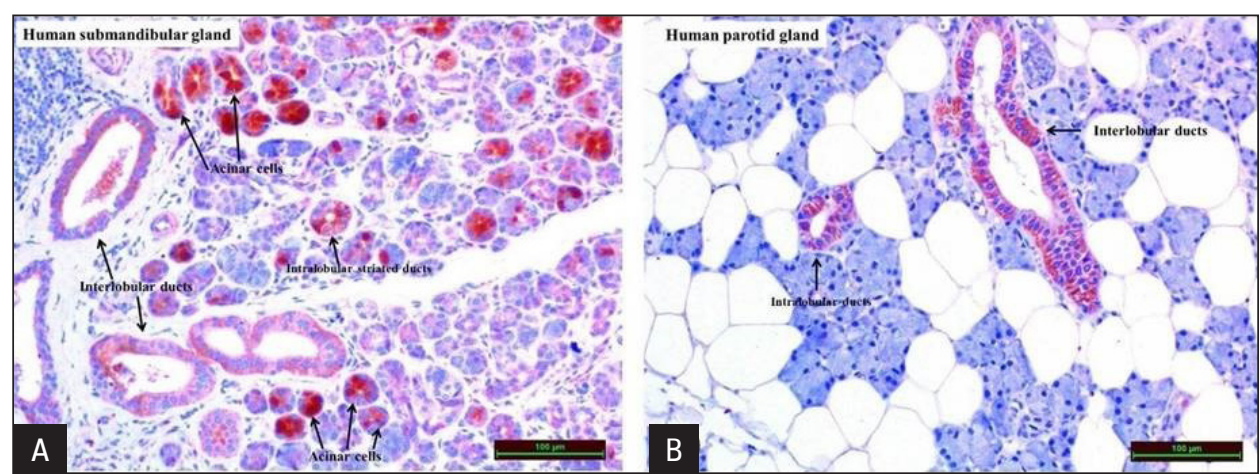

Figure 3. Asprosin immunoreactivity; intralobular ducts and interlobular ducts in the submandibular (A) and parotid (B) glands

the newly identified T2DM group compared to the healthy control group patients after age adjustment and Bonferroni correction ( $p<0.001$ ). In the newly identified T2DM group, saliva asprosin levels decreased remarkably three months post metformin treatment ( $p<0.001)$. When the healthy control group patients and the three-month treatment group were compared in terms of saliva asprosin levels, these differences were not statistically significant $(p=0.068)$. Therefore, with immunohistochemical staining for asprosin immunoreactivity and checking under light microscope, asprosin immunoreactivity was observed in the submandibular gland and acinar cells in intralobular and interlobular canals (Fig. 3A). Asprosin immunoreactivity in the parotid gland was observed in intralobular and interlobular canals (Fig. 3B). 


\section{Discussion}

Asprosin is secreted from white adipose tissues, pancreatic $\beta$-cells, and salivary glands and causes the release of hepatic glucose $[9,10,14-16]$. In the fasting state, plasma asprosin level increases, and it decreases after eating [9]. Studies in humans and animal models show that serum asprosin levels increase in patients with insulin resistance and/or T2DM [11]. In another study, it was shown that insulin sensitivity increased as a result of inhibition of asprosin with specific antibodies [9]. However, in a study by Zhang et al. serum asprosin levels were not found to be associated with other glucose metabolism parameters other than $\mathrm{HbA}_{1 \mathrm{c}}$ in individuals with normal glucose tolerance and in newly identified diabetics [11]. When all of these human and animal studies are evaluated as a whole, it can be seen that there may be a strong induction-effect relationship between T2DM and asprosin, showing that serum asprosin levels can act as an indicator for the early identification of T2DM and may also be an important hormone as a treatment goal. In light of this information, our study aimed to identify the possible correlation between asprosin and T2DM in newly identified patients and to document how this relationship was affected after metformin treatment, by measuring both plasma and saliva asprosin levels.

Previous studies have shown that plasma asprosin levels increase in the newly identified diabetic patients [11]. In our study, plasma asprosin levels were significantly increased at the time of identification compared to the control group patients (Tab. 1), and this was statistically significant. As in previous studies, saliva asprosin levels in this study were significantly higher compared to the control group patients. Our results regarding the relationship between T2DM and asprosin are compatible with previous studies. Ugur et al. showed that asprosin is also expressed from salivary glands [16]. We also supported immunohistochemically that asprosin is expressed in both submandibular and parotid glands, in support of this study (Fig. 3). The point that makes our study superior to other studies is that it revealed the possible relationship between T2DM and asprosin by simultaneously measuring both plasma and saliva asprosin levels. The simultaneous elevation of both plasma and saliva asprosin levels in individuals with new T2DM identification suggests that the relationship with T2DM may be closer than predicted. Another advantage of our study over others is that pre-treatment and post-treatment asprosin levels were measured and compared in the newly identified T2DM patients. As mentioned above, almost all treatment guidelines related to diabetes, along with lifestyle changes and dietary recommendations, recommend metformin as the first drug in treatment if there are no contraindications $[7,8]$. Guided by this information, we randomly selected our patient group among those receiving metformin therapy. There are several commercial forms of metformin, and so, to eliminate the heterogeneity between commercial forms and not to discuss the results of our study within this aspect, we selected patients from among those who used only one commercial preparation. When we evaluated the results of the study, after three months of metformin treatment, we found that both plasma and saliva asprosin levels decreased significantly compared to the pre-treatment period. When we compare the three-month post-treatment group with the healthy control group patients, we found that the previous statistical difference in terms of plasma and saliva asprosin levels disappeared. This showed that asprosin levels decreased with treatment. Although asprosin levels decreased with treatment, they did not fall below the asprosin levels of the healthy control group patients. Metformin lowers blood glucose by several mechanisms. However, glucose lowering has its greatest effect by inhibiting gluconeogenesis and decreasing hepatic glucose output [17-19]. Asprosin, on the other hand, promotes hepatic glucose production via the $G$ protein-cyclic adenosine monophosphate (cAMP) - protein kinase A (PKA) pathway, thereby increasing hepatic glucose output. In a sense, it has a protective effect from hypoglycaemia $[9,10]$. Asprosin and metformin play an important role in maintaining the glucose balance in the body, although they have an opposite effect on different pathways. But do asprosin and metformin interact via an unknown pathway in maintaining this balance? The interaction of asprosin with other appetising and glucogenic hormones in the mediation of food intake followed by hepatic glucose release is not fully understood because hormones like norepinephrine, epinephrine, glucocorticoids, and glucagon are well known to increase glucose release levels from hepatocytes [9]. It is also interesting that Romere et al. reported that asprosin treatment did not affect the above-mentioned glucogenic hormone levels [9]. It is also known as general information that glucagon can exhibit its glucogenic effects by activating the G protein-cAMP-PKA axis [9]. However, Romere et al. showed that there is no change in the glucogenic effects of asprosin on hepatocytes by inhibiting the glucagon receptor [9]. We believe that broader molecular studies are needed to elucidate these issues, although some studies suggest that asprosin may be a hypoglycaemia-protective hormone, such as a contra-regulatory hormone, although most studies show that asprosin contributes to diabetes formation [9, 10]. Returning to the results of our study, we found that after three months of treatment of the patient group with met- 
formin, $\mathrm{HbA}_{1 \mathrm{c}}$ decreased to a statistical significance compared to the pre-treatment period. Simultaneously, we found serum and saliva asprosin levels to be low. This relationship between $\mathrm{HbA}_{1 \mathrm{c}}$ and asprosin was supportive of the study conducted by Zhang et al. [11].

It is known that chronic and low-grade inflammation occurring in hyperlipidaemic conditions causes lipotoxicity in $\beta$-cells of the pancreas, and this lipotoxicity plays a causal role in $\beta$-cell dysfunction [20]. However, the mechanisms underlying $\beta$-cell dysfunction due to hyperlipidaemia have not been fully investigated. To enlighten this issue, according to the hypothesis proposed by Taeseung et al., asprosin induced by hyperlipidaemia contributed to the development of T2DM with $\beta$-cell dysfunction [15]. As assumed, they showed that glucose-induced insulin secretion was impaired, and apoptosis increases with inflammation caused by treating mouse insulinoma 6 (MIN6) and human primary islet cells with recombinant asprosin [15]. In their study, Zhang et al. displayed that as serum triglyceride levels increase, asprosin levels increase simultaneously [11]. This study signifies that triglyceride levels were higher at the time of identification in patients newly identified with T2DM in comparative identity to the control group patients, and the asprosin levels that we measured simultaneously in plasma and saliva were also high. We observed that both triglyceride levels and asprosin levels had decreased level after 90 days of treatment. In our study, there was also a similar relationship between LDL-C and asprosin. The findings we obtained were consistent with the results obtained by Zhang et al. [11] and Tang et al. [20], while Zhang et al. [11] did not find any relationship between diastolic and systolic blood pressures and asprosin levels. This study found higher diastolic and systolic blood pressures in the newly identified T2DM group patients in comparison with the healthy control group patients. Serum and saliva asprosin levels were accompanied by these high findings. These findings suggest that there may also be a link between hypertension and asprosin levels. Of course, this link must be revealed in more detail with further studies. The relationship between asprosin levels and obesity has been demonstrated in previous studies. For example, Xinyue et al. [21] showed that asprosin levels increase as the body mass index (BMI) increases numerically although there is no statistical difference in terms of BMI between the groups. In another study, which included 117 obese people with BMI $>35 \mathrm{~kg} / \mathrm{m}^{2}$ and 57 healthy individuals, fasting asprosin levels displayed a significantly higher level in obese patients than in healthy patients [22]. Because there was no statistical difference in terms of BMI between the groups in our study, we did not consider it appropriate to make a comparison within this aspect.
Our study has several important limitations. The first of these is the small number of cases. The second is that the patient follow-up times are short. The third is that our post-treatment evaluations were made only in patients using metformin, and the fourth is the mean age of our patients, and that the healthy control group patients were different from each other. However, despite these limitations, considering our pre- and post-treatment evaluations, our study is the first study in this sense. However, we believe that studies with a larger number of patients and longer follow-up are needed to improve these evaluations.

\section{Conclusion}

This study showed that serum and saliva asprosin levels significantly increased in newly identified individuals with T2DM. The data of this study show that asprosin could be a potential risk factor related to the development of T2DM. In light of these data, we think that serum and saliva asprosin level measurements are an important indicator for predicting the development of diabetes, and this hormone can be evaluated as a target molecule to treat diabetes.

\section{Conflicts of interest}

The authors declare that there are no conflicts of interest concerning the publication of this article.

\section{Acknowledgments}

The authors acknowledge Tuncay Kuloglu of the Frrat University Pathology Department for donating salivary glands and testing immunohistochemical staining and evaluation of the glands.

\section{References}

1. Wang CCi, Shah ACC. (eds). Medical Management of Type 1 Diabetes. 7th ed. American Diabetes Association, Alexandria 2017.

2. American Diabetes Association. Diagnosis and classification of diabetes mellitus. Diabetes Care. 2014; 37 Suppl 1: S81-S90, doi: 10.2337/dc14-S081, indexed in Pubmed: 24357215.

3. Saeedi P, Petersohn I, Salpea P, et al. IDF Diabetes Atlas Committee. Global and regional diabetes prevalence estimates for 2019 and projections for 2030 and 2045: Results from the International Diabetes Federation Diabetes Atlas, 9 edition. Diabetes Res Clin Pract. 2019; 157: 107843 , doi: 10.1016/j.diabres.2019.107843, indexed in Pubmed: 31518657.

4. Zhou T, Xu X, Du M, et al. A preclinical overview of metformin for the treatment of type 2 diabetes. Biomed Pharmacother. 2018; 106: 1227-1235, doi: 10.1016/j.biopha.2018.07.085, indexed in Pubmed: 30119191.

5. Baron AD, Schaeffer L, Shragg P, et al. Role of hyperglucagonemia in maintenance of increased rates of hepatic glucose output in type II diabetics. Diabetes. 1987; 36(3): 274-283, doi: 10.2337/diab.36.3.274, indexed in Pubmed: 2879757.

6. Molina Vega M, Muñoz-Garach A, Tinahones FJ. Pharmacokinetic drug evaluation of exenatide for the treatment of type 2 diabetes. Expert Opin Drug Metab Toxicol. 2018; 14(2): 207-217, doi: 10.1080/17425255.2018.14 20160, indexed in Pubmed: 29260924.

7. Davies MJ, D'Alessio DA, Fradkin J, et al. Management of Hyperglycemia in Type 2 Diabetes, 2018. A Consensus Report by the American Diabetes Association (ADA) and the European Association for the Study of Diabetes (EASD). Diabetes Care. 2018; 41(12): 2669-2701, doi: 10.2337/dci18-0033, indexed in Pubmed: 30291106. 
8. American Diabetes Association. 9. Pharmacologic Approaches to Glycemic Treatment. Diabetes Care. 2019; 42(Suppl 1): S90-S9S102, doi: 10.2337/dc19-S009, indexed in Pubmed: 30559235.

9. Romere C, Duerrschmid C, Bournat J, et al. Asprosin, a Fasting-Induced Glucogenic Protein Hormone. Cell. 2016; 165(3): 566-579, doi: 10.1016/j. cell.2016.02.063, indexed in Pubmed: 27087445.

10. Greenhill C. Liver: Asprosin - new hormone involved in hepatic glucose release. Nat Rev Endocrinol. 2016; 12(6): 312, doi: 10.1038/nrendo.2016.66, indexed in Pubmed: 27125501.

11. Zhang L, Chen C, Zhou N, et al. Circulating asprosin concentrations are increased in type 2 diabetes mellitus and independently associated with fasting glucose and triglyceride. Clin Chim Acta. 2019; 489: 183-188, doi: 10.1016/j.cca.2017.10.034, indexed in Pubmed: 29104036

12. Gillett MJ, Kilpatrick ES, Bloomgarden ZT, et al. International Expert Committee. International Expert Committee report on the role of the A1C assay in the diagnosis of diabetes. Diabetes Care. 2009; 32(7): 1327-1334, doi: 10.2337/dc09-9033, indexed in Pubmed: 19502545 .

13. Aydin S. A short history, principles, and types of ELISA, and our laboratory experience with peptide/protein analyses using ELISA. Peptides. 2015; 72: 4-15, doi: 10.1016/j.peptides.2015.04.012, indexed in Pubmed: 25908411.

14. Kajimura S. Adipose tissue in 2016: Advances in the understanding of adipose tissue biology. Nat Rev Endocrinol. 2017; 13(2): 69-70, doi: 10.1038/nrendo.2016.211, indexed in Pubmed: 28051117.

15. Lee $\mathrm{T}$, Yun $\mathrm{S}$, Jeong $\mathrm{JiH}$, et al. Asprosin impairs insulin secretion in response to glucose and viability through TLR4/JNK-mediated in- flammation. Mol Cell Endocrinol. 2019; 486: 96-104, doi: 10.1016/j. mce.2019.03.001, indexed in Pubmed: 30853600.

16. Ugur K, Aydin S. Saliva and Blood Asprosin Hormone Concentration Associated with Obesity. Int J Endocrinol. 2019; 2019: 2521096 doi: 10.1155/2019/2521096, indexed in Pubmed: 31049060.

17. Fullerton MD, Galic S, Marcinko K, et al. Single phosphorylation sites in Acc1 and Acc2 regulate lipid homeostasis and the insulin-sensitizing effects of metformin. Nat Med. 2013; 19(12): 1649-1654, doi: 10.1038/nm.3372, indexed in Pubmed: 24185692.

18. Shaw RJ. Metformin trims fats to restore insulin sensitivity. Nat Med. 2013; 19(12): 1570-1572, doi: 10.1038/nm.3414, indexed in Pubmed: 24309653.

19. Shaw RJ, Lamia KA, Vasquez D, et al. The kinase LKB1 mediates glucose homeostasis in liver and therapeutic effects of metformin. Science. 2005; 310(5754): 1642-1646, doi: 10.1126/science.1120781, indexed in Pubmed: 16308421.

20. Tang C, Naassan A, Chamson-Reig A, et al. Susceptibility to Fatty Acid-Induced $\beta$-Cell Dysfunction Is Enhanced in Prediabetic Diabetes-Prone BioBreeding Rats: A Potential Link Between $\beta$-Cell Lipotoxicity and Islet Inflammation. Endocrinology. 2013; 154(1): 89-101, doi: 10.1210/en.2012-1720, indexed in Pubmed: 23150493.

21. Zhang $X$, Jiang $H$, Ma X, et al. Increased serum level and impaired response to glucose fluctuation of asprosin is associated with type 2 diabetes mellitus. J Diabetes Investig. 2020; 11(2): 349-355, doi: 10.1111/jdi.13148, indexed in Pubmed: 31529619.

22. Wang CY, Lin TA, Liu KH, et al. Serum asprosin levels and bariatric surgery outcomes in obese adults. Int J Obes (Lond). 2019; 43(5): 1019-1025, doi: 10.1038/s41366-018-0248-1, indexed in Pubmed: 30459402. 